\title{
Dwork cohomology, de Rham cohomology, and hypergeometric functions
}

\author{
Alan Adolphson* \\ Department of Mathematics \\ Oklahoma State University \\ Stillwater, Oklahoma 74078 \\ adolphs@math.okstate.edu
}

\author{
Steven Sperber \\ School of Mathematics \\ University of Minnesota \\ Minneapolis, Minnesota 55455 \\ sperber@math.umn.edu
}

Dedicated to Hugh Turrittin on the occasion of his ninetieth birthday

\begin{abstract}
In the 1960's, Dwork developed a $p$-adic cohomology theory of de Rham type for varieties over finite fields, based on a trace formula for the action of a Frobenius operator on certain spaces of $p$-analytic functions. One can consider a purely algebraic analogue of Dwork's theory for varieties over a field of characteristic zero and ask what is the connection between this theory and ordinary de Rham cohomology. Katz 13, 14] showed that Dwork cohomology coincides with the primitive part of de Rham cohomology for smooth projective hypersurfaces, but the exact relationship for varieties of higher codimension has been an open question. In this article, we settle the case of smooth, affine, complete intersections.
\end{abstract}

\section{$\S 1$. Introduction}

We refer the reader to Katz[15 for general information on connections, de Rham cohomology, and the Gauss-Manin connection. Some of this material will be reviewed in section 2. A convenient reference for the properties of smooth schemes that we use is Altman-Kleiman[4, Chap. VII, sect. 5]. Let $S$ be a smooth, equidimensional C-scheme and $X$ a smooth, equidimensional $S$-scheme of relative dimension $N$. Let $Y \subseteq X$ be a smooth, closed $S$-subscheme, purely of codimension $r$. Let $\mathcal{E}$ be a locally free $\mathcal{O}_{X}$-module of finite rank with an integrable $\mathbf{C}$-connection

$$
\nabla: \mathcal{E} \rightarrow \Omega_{X / \mathbf{C}}^{1} \bigotimes_{\mathcal{O}_{X}} \mathcal{E}
$$

Let $j: Y \hookrightarrow X$ be the inclusion and let

$$
\nabla_{Y}: j^{*}(\mathcal{E}) \rightarrow \Omega_{Y / \mathbf{C}}^{1} \bigotimes_{\mathcal{O}_{Y}} j^{*}(\mathcal{E})
$$

be the pullback of $\nabla$ to a connection on $j^{*}(\mathcal{E})$. We consider the problem of computing the Gauss-Manin connection (an integrable $\mathbf{C}$-connection) on the de Rham cohomology sheaves

$$
H_{\mathrm{DR}}^{n}\left(Y / S,\left(j^{*}(\mathcal{E}), \nabla_{Y}\right)\right),
$$

the hypercohomology of the functor "direct image under the map $Y \rightarrow S$ " with respect to the complex $\Omega_{Y / S} \bigotimes_{\mathcal{O}_{Y}} j^{*}(\mathcal{E})$.

In this article, we treat the case where $S, X, Y$ are affine. Let $X=\operatorname{Spec}(A)$ and let $f_{1}, \ldots, f_{r} \in A$. Let $y_{1}, \ldots, y_{r}$ be indeterminates and consider

$$
\mathbf{A}_{X}^{r}=\operatorname{Spec}\left(A\left[y_{1}, \ldots, y_{r}\right]\right)
$$

*Partially supported by NSF grant no. DMS-9305514. 
with projection $\pi: \mathbf{A}_{X}^{r} \rightarrow X$. Put

$$
F=\sum_{j=1}^{r} y_{j} f_{j} \in A\left[y_{1}, \ldots, y_{r}\right] .
$$

Let $\nabla_{\mathbf{A}_{X}^{r}}$ be the pullback of $\nabla$ to a connection on $\pi^{*}(\mathcal{E})$. We let $\nabla_{F}$ be the "twist" of $\nabla_{\mathbf{A}_{X}^{r}}$ by $\exp F$, i. e.,

$$
\nabla_{F}(e)=\nabla_{\mathbf{A}_{X}^{r}}(e)+d_{\mathbf{A}_{X}^{r} / \mathbf{C}} F \otimes e,
$$

where $e$ is a section of $\pi^{*}(\mathcal{E})$ over an open subset of $\mathbf{A}_{X}^{r}$. We say that $f_{1}, \ldots, f_{r} \in A$ define a smooth complete intersection $Y \subseteq X$ if $Y=\operatorname{Spec}\left(A /\left(f_{1}, \ldots, f_{r}\right)\right)$ is a smooth $S$-scheme of codimension $r$ in $X$. In particular, $Y$ is regularly immersed in $X$.

Our main result is the following.

Theorem 1.1 Suppose $f_{1}, \ldots, f_{r}$ define a smooth complete intersection $Y \subseteq X$. Then for every $n \in \mathbf{Z}$ there is an isomorphism of $\mathcal{O}_{S}$-modules with $\mathbf{C}$-connections

$$
H_{\mathrm{DR}}^{n}\left(Y / S,\left(j^{*}(\mathcal{E}), \nabla_{Y}\right)\right) \simeq H_{\mathrm{DR}}^{n+2 r}\left(\mathbf{A}_{X}^{r} / S,\left(\pi^{*}(\mathcal{E}), \nabla_{F}\right)\right) .
$$

For example, suppose $S=\operatorname{Spec}(R)$ and $X=\mathbf{A}_{R}^{N}$. Let $\mathcal{E}=\mathcal{O}_{X}$ with the standard connection, so that $j^{*}(\mathcal{E})=\mathcal{O}_{Y}$ with the standard connection. Then the left-hand side is just the de Rham cohomology $H_{\mathrm{DR}}^{n}(Y / S)$ of the smooth complete intersection $Y \subseteq \mathbf{A}_{R}^{N}$ defined by $f_{1}, \ldots, f_{r} \in R\left[x_{1}, \ldots, x_{N}\right]$. We consider the top-dimensional cohomology group. Put $C=R\left[x_{1}, \ldots, x_{N}, y_{1}, \ldots, y_{r}\right]$, the coordinate ring of $\mathbf{A}_{X}^{r}$. Writing out the connection $\nabla_{F}$ in local coordinates, we see that the theorem implies

$$
H_{\mathrm{DR}}^{N-r}(Y / S) \simeq C /\left(\sum_{i=1}^{N} D_{x_{i}}(C)+\sum_{j=1}^{r} D_{y_{i}}(C)\right)
$$

where

$$
\begin{aligned}
D_{x_{i}} & =\frac{\partial}{\partial x_{i}}+\sum_{j=1}^{r} y_{j} \frac{\partial f_{j}}{\partial x_{i}} \\
D_{y_{j}} & =\frac{\partial}{\partial y_{j}}+f_{j} .
\end{aligned}
$$

Furthermore, for $\partial \in \operatorname{Der}_{\mathbf{C}}(R)$, the action of $\partial$ on $H_{\mathrm{DR}}^{N-r}(Y / S)$ via the Gauss-Manin connection is identified under this isomorphism with the action induced on the right-hand side of (1.2) by the action of

$$
D_{\partial}=\partial+\sum_{j=1}^{r} y_{j} f_{j}^{\partial}
$$

on $C$, where $\partial$ acts on elements of $C$ (i. e., polynomials over $R$ ) by acting on their coefficients. (In particular, $f_{j}^{\partial}$ denotes the polynomial obtained from $f_{j}$ by applying $\partial$ to its coefficients.)

In general, by allowing one to replace $Y$ by $\mathbf{A}_{X}^{r}$ and thus work more globally, the theorem makes the Gauss-Manin connection easier to compute. As an application, we show that periods of differential forms on smooth complete intersections in $\mathbf{A}_{R}^{N}$ satisfy hypergeometric differential equations. Specifically, we consider a spanning set of cohomology classes of $H_{\mathrm{DR}}^{N-r}(Y / S)$ and for each of these cohomology classes we construct a left ideal in the ring of differential operators on $R$ that annihilates it. This construction gives a new procedure for computing Fuchs-Picard equations of complete intersections.

Remark. The statement of Theorem 1.1 makes sense only in the affine case, where one has global regular functions $f_{1}, \ldots, f_{r}$ defining the subvariety $Y$ and the connection $\nabla_{F}$. However, even when $X$ is not affine, an analogue of Theorem 4.5 below remains valid. In this context, our result is closely related to a theorem of Hartshorne 12, Chapter III, Theorem 8.1]. We plan to return to this topic in a future article. The affine case is sufficient for the application to hypergeometric differential equations treated here.

The cohomology groups on the right-hand side in Theorem 1.1 are the "Dwork cohomology" referred to in the title of this article. The first result in the direction of Theorem 1.1 was proved by Katz[13, 14]. Motivated 
by Dwork's calculations [8], which showed that the deformation equation in Dwork's p-adic cohomology theory was identical to the corresponding Fuchs-Picard equation, Katz proved that Dwork cohomology coincides with the primitive part of de Rham cohomology for smooth projective hypersurfaces. It was an open question to determine the relation between these two cohomologies for smooth complete intersections of codimension greater than one. This is what we accomplish here.

This paper is organized as follows. In section 2, we briefly review some properties of connections. In section 3, we use the Leray spectral sequence of the composition $\mathbf{A}_{X}^{r} \rightarrow X \rightarrow S$ to reduce Theorem 1.1 to Theorem 4.5. Theorem 4.5 is then proved in sections 4 and 5 . In section 6 , we show that de Rham cohomology classes on a complete intersection satisfy hypergeometric differential equations.

The results of this article have been generalized by Dimca et al. []. Using the theory of $\mathcal{D}$-modules, they obtain stronger results while avoiding some of the more computational aspects of our approach.

\section{$\S 2 . \quad$ Connections}

We review some basic properties of connections on affine schemes. Let $S=\operatorname{Spec}(R)$, where $R$ is a smooth C-algebra and $X=\operatorname{Spec}(A)$, where $A$ is a smooth $R$-algebra. Let $Y \subseteq X$ be a smooth, closed $S$-subvariety, say, $Y=\operatorname{Spec}(B)$ with $B=A / I$. We use algebraic rather than geometric notation, e. g., we write $\Omega_{A / R}^{1}$ rather than $\Omega_{X / S}^{1}$. Let $M$ be an $A$-module with a $\mathbf{C}$-connection $\nabla$, i. e., a homomorphism of abelian groups

$$
\nabla: M \rightarrow \Omega_{A / \mathbf{C}}^{1} \bigotimes_{A} M
$$

satisfying

$$
\nabla(a m)=a \nabla(m)+d_{A / \mathbf{C}} a \otimes m
$$

for all $a \in A, m \in M$, where $d_{A / \mathbf{C}}$ denotes the exterior derivative. It is also convenient to think of a C-connection as an $A$-linear map

$$
\nabla: \operatorname{Der}_{\mathbf{C}}(A) \rightarrow \operatorname{End}_{\mathbf{C}}(M)
$$

where $\operatorname{Der}_{\mathbf{C}}(A)$ denotes the Lie algebra of $\mathbf{C}$-linear derivations of $A$. Let $M_{B}=M \bigotimes_{A} B$. We define a connection on $M_{B}$ by pullback. Specifically, tensoring (2.1) with $B$ gives a map

$$
M_{B} \rightarrow \Omega_{A / \mathbf{C}}^{1} \bigotimes_{A} M_{B} \simeq\left(\Omega_{A / \mathbf{C}}^{1} \bigotimes_{A} B\right) \bigotimes_{B} M_{B}
$$

The natural map $\Omega_{A / \mathbf{C}}^{1} \otimes_{A} B \rightarrow \Omega_{B / \mathbf{C}}^{1}$ induces

$$
\left(\Omega_{A / \mathrm{C}}^{1} \bigotimes_{A} B\right) \bigotimes_{B} M_{B} \rightarrow \Omega_{B / \mathrm{C}}^{1} \bigotimes_{B} M_{B}
$$

The composition of (2.2) and (2.3) defines a $\mathbf{C}$-connection on the $B$-module $M_{B}$, which we denote by $\nabla_{B}$.

When $\nabla$ is an integrable connection, there is an associated de Rham complex $\Omega_{A / \mathbf{C}} \otimes_{A} M$ whose differential we denote by the same symbol used for the connection. For later use, we recall that

$$
\nabla: \Omega_{A / \mathbf{C}}^{n} \bigotimes_{A} M \rightarrow \Omega_{A / \mathbf{C}}^{n+1} \bigotimes_{A} M
$$

is the homomorphism of abelian groups given by

$$
\nabla(\omega \otimes m)=d_{A / \mathbf{C}} \omega \otimes m+(-1)^{n} \omega \wedge \nabla(m),
$$

where $\omega \in \Omega_{A / \mathbf{C}}^{n}, m \in M$, and $\omega \wedge \nabla(m)$ denotes the image of $\omega \otimes \nabla(m)$ under the canonical map

$$
\Omega_{A / \mathbf{C}}^{n} \bigotimes_{A}\left(\Omega_{A / \mathbf{C}}^{1} \bigotimes_{A} M\right) \rightarrow \Omega_{A / \mathbf{C}}^{n+1} \bigotimes_{A} M
$$


A C-connection on $M$ is, in a natural way, an $R$-connection via the map $\Omega_{A / \mathbf{C}}^{1} \rightarrow \Omega_{A / R}^{1}$ (or, equivalently, via the inclusion $\left.\operatorname{Der}_{R}(A) \subseteq \operatorname{Der}_{\mathbf{C}}(A)\right)$. By abuse of notation, we also denote the $R$-connection by $\nabla$. Furthermore, if $\nabla$ is an integrable connection, then so is $\nabla_{B}$. In this case, $\nabla$ (resp. $\nabla_{B}$ ) gives rise to an associated de Rham complex $\left(\Omega_{A / R}^{*} \bigotimes_{A} M, \nabla\right)$ (resp. $\left.\left(\Omega_{B / R} \bigotimes_{B} M_{B}, \nabla_{B}\right)\right)$. We denote by $H_{\mathrm{DR}}^{n}(A / R,(M, \nabla))$ (resp. $H_{\mathrm{DR}}^{n}\left(B / R,\left(M_{B}, \nabla_{B}\right)\right)$ ) the cohomology groups of this complex. We wish to compute the $H_{\mathrm{DR}}^{n}\left(B / R,\left(M_{B}, \nabla_{B}\right)\right)$ and their associated Gauss-Manin connections when $M$ is projective of finite rank. (We recall that the elements of $\operatorname{Der}_{\mathbf{C}}(R)$ act on this cohomology group via the Gauss-Manin connection, which is integrable.)

Let $y_{1}, \ldots, y_{r}$ be additional variables and consider $\mathbf{A}_{A}^{r}$ with coordinate $\operatorname{ring} C=A\left[y_{1}, \ldots, y_{r}\right]$. Let $f_{1}, \ldots, f_{r} \in A$ and put

$$
F=\sum_{j=1}^{r} y_{j} f_{j} \in C
$$

Put $M_{C}=M \bigotimes_{A} C$ and define a $\mathbf{C}$-connection $\nabla_{C}$ on the $C$-module $M_{C}$ by pullback as before. We define another connection $\nabla_{F}$ on $M_{C}$ by twisting with $\exp F$. Specifically, for $\mu \in M_{C}$, we put

$$
\nabla_{F}(\mu)=\nabla_{C}(\mu)+d_{C / \mathbf{C}} F \otimes \mu
$$

As before, these define $R$-connections as well, and when $\nabla$ is integrable, so are $\nabla_{C}$ and $\nabla_{F}$. We consider the complex $\left(\Omega_{C / R} \otimes_{C} M_{C}, \nabla_{F}\right)$ and its cohomology groups $H_{\mathrm{DR}}^{n}\left(C / R,\left(M_{C}, \nabla_{F}\right)\right)$.

Now suppose that $f_{1}, \ldots, f_{r}$ define a smooth complete intersection $Y \subseteq X$, i. e., $Y=\operatorname{Spec}(A / I)$, $I=\left(f_{1}, \ldots, f_{r}\right)$, is a smooth $R$-scheme of codimension $r$ in $X$. Theorem 1.1 can be restated in the following form.

Theorem 2.5 Suppose $f_{1}, \ldots, f_{r}$ define a smooth complete intersection $Y \subseteq X$ and $M$ is a projective Amodule of finite rank. Then for every $n \in \mathbf{Z}$ there is an isomorphism of $R$-modules with $\mathbf{C}$-connections

$$
H_{\mathrm{DR}}^{n}\left(B / R,\left(M_{B}, \nabla_{B}\right)\right) \simeq H_{\mathrm{DR}}^{n+2 r}\left(C / R,\left(M_{C}, \nabla_{F}\right)\right)
$$

\section{$\S 3 . \quad$ Leray spectral sequence}

The purpose of this section is to describe the relation between the cohomology of the complexes $\Omega_{C / R} \otimes_{C} M_{C}$ and $\Omega_{C / A} \otimes_{C} M_{C}$, both with connection $\nabla_{F}$. We denote the cohomology groups of the latter complex by $H_{\mathrm{DR}}^{n}\left(C / A,\left(M_{C}, \nabla_{F}\right)\right)$. They are $A$-modules with a $\mathbf{C}$-connection (the Gauss-Manin connection), which we denote by $\delta$. There is a Leray spectral sequence ([15, Remark (3.3)])

$$
E_{2}^{p, q}=H_{\mathrm{DR}}^{p}\left(A / R,\left(H_{\mathrm{DR}}^{q}\left(C / A,\left(M_{C}, \nabla_{F}\right)\right), \delta\right)\right) \Rightarrow H_{\mathrm{DR}}^{p+q}\left(C / R,\left(M_{C}, \nabla_{F}\right)\right)
$$

Theorem 3.2 Suppose $f_{1}, \ldots, f_{r}$ define a smooth complete intersection $Y \subseteq X$ and $M$ is a projective Amodule of finite rank. Then

$$
H_{\mathrm{DR}}^{q}\left(C / A,\left(M_{C}, \nabla_{F}\right)\right)=0 \quad \text { for } q \neq r,
$$

hence the Leray spectral sequence (3.1) collapses and we get isomorphisms of $R$-modules with $\mathbf{C}$-connections

$$
H_{\mathrm{DR}}^{n}\left(A / R,\left(H_{\mathrm{DR}}^{r}\left(C / A,\left(M_{C}, \nabla_{F}\right)\right), \delta\right)\right) \simeq H_{\mathrm{DR}}^{n+r}\left(C / R,\left(M_{C}, \nabla_{F}\right)\right) \quad \text { for all } n .
$$

Before beginning the proof, we describe the action of $\nabla_{F}$ on $\Omega_{C / A} \otimes_{C} M_{C}$ in terms of the local coordinates $y_{1}, \ldots, y_{r}$. An element of $\Omega_{C / A}^{n} \bigotimes_{C} M_{C}$ can be written uniquely as a sum of elements of the form

$$
\omega=\mu d y_{j_{1}} \wedge \cdots \wedge d y_{j_{n}}
$$

with $\mu \in M_{C}$. Then

$$
\nabla_{F}(\omega)=\left(\sum_{j=1}^{r} D_{y_{j}}(\mu) d y_{j}\right) \wedge d y_{j_{1}} \wedge \cdots \wedge d y_{j_{n}}
$$


where $D_{y_{j}}$ is given by (1.4). Of course, $\mu \in M_{C}$ can be uniquely written as a sum of elements of the form $m y_{1}^{a_{1}} \cdots y_{r}^{a_{r}}$ with $m \in M$, and we have

$$
D_{y_{j}}\left(m y_{1}^{a_{1}} \cdots y_{r}^{a_{r}}\right)=a_{j} m y_{j}^{-1} y_{1}^{a_{1}} \cdots y_{r}^{a_{r}}+f_{j} m y_{1}^{a_{1}} \cdots y_{r}^{a_{r}} .
$$

We now define a grading and filtration on $M_{C}$ that will be used throughout this article. An element of $M_{C}$ can be uniquely written as a sum of elements of the form $m y_{1}^{a_{1}} \cdots y_{r}^{a_{r}}$ with $m \in M$. We define

$$
\operatorname{deg}\left(m y_{1}^{a_{1}} \cdots y_{r}^{a_{r}}\right)=a_{1}+\cdots+a_{r}
$$

Let $M_{C}^{(d)} \subseteq M_{C}$ be the $R$-span of elements $m y^{a}, m \in M$, with $\operatorname{deg}\left(m y^{a}\right)=d$. The corresponding (increasing) filtration $F$. on $M_{C}$ is defined by letting $F_{d} M_{C}$ be the $R$-span of those elements $m y^{a}$ of degree $\leq d$.

Proof of Theorem 3.2. We extend the filtration $F$. to $\Omega_{C / A} \otimes_{C} M_{C}$ by defining

$$
F_{d}\left(\Omega_{C / A} \bigotimes_{C} M_{C}\right)=\Omega_{C / A}^{\cdot} \bigotimes_{C} F_{d} M_{C}
$$

To prove the vanishing result (3.3), it suffices to prove the corresponding result for the associated graded complex relative to this filtration. By (3.4) and (3.5), the associated graded complex is just the Koszul complex on $M_{C}$ defined by $f_{1}, \ldots, f_{r}$. This Koszul complex obviously decomposes into a direct sum over $\left(a_{1}, \ldots, a_{r}\right) \in \mathbf{N}^{r}$ of copies of the Koszul complex on $M$ defined by $f_{1}, \ldots, f_{r}$. Since projective modules are locally free and cohomology commutes with localization, we may assume that $M$ is a free $A$-module of finite rank. We are thus reduced to proving the following.

Lemma 3.6 Let $\operatorname{Kos}\left(A ; f_{1}, \ldots, f_{r}\right)$ be the Koszul complex on $A$ defined by $f_{1}, \ldots, f_{r}$. Then

$$
H^{n}\left(\operatorname{Kos}\left(A ; f_{1}, \ldots, f_{r}\right)\right)=0 \quad \text { for } n \neq r .
$$

Proof. The cohomology groups of this Koszul complex are $A$-modules and cohomology commutes with localization, so it suffices to prove the corresponding vanishing of cohomology for each of the local rings $A_{\mathbf{p}}$, where $\mathbf{p}$ is a maximal ideal of $A$. Since these cohomology groups are supported on $Y$ (17, Theorem 16.4]), we may assume $\mathbf{p}$ corresponds to a point of $Y$. But the smooth complete intersection hypothesis implies that for such $\mathbf{p}$, the images of $f_{1}, \ldots, f_{r}$ in $A_{\mathbf{p}}$ form a regular sequence. The assertion then follows from well-known properties of regular sequences.

Corollary 3.7 Suppose $f_{1}, \ldots, f_{r}$ define a smooth complete intersection $Y \subseteq X$ and $M$ is a projective $A$ module of finite rank. Suppose $\mu_{j} \in F_{d} M_{C}, j=1, \ldots, r$, are such that $\sum_{j=1}^{r} D_{y_{j}}\left(\mu_{j}\right) \in F_{d-1} M_{C}$. Then there exist $\mu_{j}^{\prime} \in F_{d-1} M_{C}, j=1, \ldots, r$, such that $\sum_{j=1}^{r} D_{y_{j}}\left(\mu_{j}^{\prime}\right)=\sum_{j=1}^{r} D_{y_{j}}\left(\mu_{j}\right)$.

Proof. Let $\mu_{j}^{(d)}$ denote the homogeneous component of degree $d$ of $\mu_{j}$. The hypothesis implies that $\sum_{j=1}^{r} f_{j} \mu_{j}^{(d)}=0$, i. e., $\sum_{j=1}^{r}(-1)^{j-1} \mu_{j}^{(d)} d y_{1} \wedge \cdots \wedge \widehat{d y_{j}} \wedge \cdots \wedge d y_{r}$ is an $(r-1)$-cocycle in $\operatorname{Kos}\left(M_{C} ; f_{1}, \ldots, f_{r}\right)$. But we have just seen that the $(r-1)$-st cohomology group of this complex vanishes, hence there exists a skew-symmetric set $\left\{\eta_{i j}\right\}_{i, j=1}^{r}$ (i. e., $\eta_{j i}=-\eta_{i j}$ ), with $\eta_{i j} \in M_{C}^{(d)}$, such that $\mu_{j}^{(d)}=\sum_{k=1}^{r} f_{k} \eta_{j k}$. Define $\mu_{j}^{\prime}=\mu_{j}-\sum_{k=1}^{r} D_{y_{k}}\left(\eta_{j k}\right)$. Then $\mu_{j}^{\prime} \in F_{d-1} M_{C}$ and $\sum_{j=1}^{r} D_{y_{j}}\left(\mu_{j}^{\prime}\right)=\sum_{j=1}^{r} D_{y_{j}}\left(\mu_{j}\right)$ by the skew-symmetry of $\left\{\eta_{i j}\right\}$.

\section{$\S 4$. The fundamental quasi-isomorphism}

To simplify notation, we put

$$
\bar{M}=H_{\mathrm{DR}}^{r}\left(C / A,\left(M_{C}, \nabla_{F}\right)\right),
$$

an $A$-module with the $\mathbf{C}$-connection $\delta$. Concretely, by (3.4) we identify

$$
\bar{M}=M_{C} / \sum_{j=1}^{r} D_{y_{j}}\left(M_{C}\right)
$$


The connection $\delta$ can be described explicitly as follows. For $\mu \in M_{C}$, let $[\mu]$ denote its image in $\bar{M}$. Let $m \in M$ and write

$$
\nabla(m)=\sum_{k} \omega_{k} \otimes m_{k}
$$

with $\omega_{k} \in \Omega_{A / R}^{1}$ and $m_{k} \in M$. From the definition of $\nabla_{F}$ we get

$$
\delta\left(\left[m y_{1}^{a_{1}} \cdots y_{r}^{a_{r}}\right]\right)=\sum_{k} \omega_{k} \otimes\left[m_{k} y_{1}^{a_{1}} \cdots y_{r}^{a_{r}}\right]+\sum_{j=1}^{r} d_{A / R} f_{j} \otimes\left[y_{j} m y_{1}^{a_{1}} \cdots y_{r}^{a_{r}}\right] .
$$

More generally, by (2.4), the map $\delta: \Omega_{A / R}^{n} \bigotimes_{A} \bar{M} \rightarrow \Omega_{A / R}^{n+1} \bigotimes_{A} \bar{M}$ is given by

$$
\begin{aligned}
\delta\left(\omega \otimes\left[m y_{1}^{a_{1}} \cdots y_{r}^{a_{r}}\right]\right)= & d_{A / R} \omega \otimes\left[m y_{1}^{a_{1}} \cdots y_{r}^{a_{r}}\right]+(-1)^{n} \sum_{k}\left(\omega \wedge \omega_{k}\right) \otimes\left[m_{k} y_{1}^{a_{1}} \cdots y_{r}^{a_{r}}\right]+ \\
& (-1)^{n} \sum_{j=1}^{r}\left(\omega \wedge d_{A / R} f_{j}\right) \otimes\left[y_{j} m y_{1}^{a_{1}} \cdots y_{r}^{a_{r}}\right] .
\end{aligned}
$$

Theorem 3.2 gives

$$
H_{\mathrm{DR}}^{n}(A / R,(\bar{M}, \delta)) \simeq H_{\mathrm{DR}}^{n+r}\left(C / R,\left(M_{C}, \nabla_{F}\right)\right) .
$$

In view of this isomorphism, Theorem 2.5 is an immediate consequence of the following.

Theorem 4.5 Suppose $f_{1}, \ldots, f_{r}$ define a smooth complete intersection $Y \subseteq X$ and $M$ is a projective $A$ module of finite rank. Then there is a quasi-isomorphism

$$
\bar{\Phi}:\left(\Omega_{B / R} \bigotimes_{B} M_{B}, \nabla_{B}\right) \rightarrow\left(\Omega_{A / R}^{\cdot}[-r] \bigotimes_{A} \bar{M}, \delta\right)
$$

that induces isomorphisms of $R$-modules with $\mathbf{C}$-connections

$$
H_{\mathrm{DR}}^{n}\left(B / R,\left(M_{B}, \nabla_{B}\right)\right) \simeq H_{\mathrm{DR}}^{n+r}(A / R,(\bar{M}, \delta)) \quad \text { for all } n .
$$

The proof of Theorem 4.5 will occupy the remainder of this section and the next section. In this section, we define $\bar{\Phi}$, check that it respects the $\mathbf{C}$-connections, and show that it is an isomorphism onto a subcomplex $L^{\cdot}[-r]$ of $\Omega_{A / R}^{\cdot}[-r] \bigotimes_{A} \bar{M}$. In section 5 , we prove that the inclusion $L^{\cdot} \hookrightarrow \Omega_{A / R}^{\cdot} \bigotimes_{A} \bar{M}$ is a quasi-isomorphism.

We begin by extending the filtration $F$. on $M_{C}$ defined in the previous section to the complex $\Omega_{A / R} \otimes_{A} \bar{M}$. Since $\bar{M}$ is a quotient of $M_{C}$, we get an induced filtration on $\bar{M}$ which we denote by $F$. also. Note that by Corollary 3.7 there is a natural identification

$$
F_{d} \bar{M}=F_{d} M_{C} / \sum_{j=1}^{r} D_{y_{j}}\left(F_{d} M_{C}\right)
$$

In what follows, we often make this identification without comment. We define the filtration $F$. on $\Omega_{A / R}^{\cdot} \otimes_{A} \bar{M}$ by setting

$$
F_{d}\left(\Omega_{A / R}^{n} \bigotimes_{A} \bar{M}\right)=\Omega_{A / R}^{n} \bigotimes_{A} F_{d+n-N} \bar{M}
$$

where we denote by $N$ the relative dimension of $\operatorname{Spec}(A)$ over $\operatorname{Spec}(R)$. In particular, $\Omega_{A / R}^{1}$ is locally free of rank $N$ and the complex $\Omega_{A / R} \otimes_{A} \bar{M}$ has length $N$. The indexing is chosen so that $\delta$ respects the filtration, i. e.,

$$
\delta\left(F_{d}\left(\Omega_{A / R}^{n} \bigotimes_{A} \bar{M}\right)\right) \subseteq F_{d}\left(\Omega_{A / R}^{n+1} \bigotimes_{A} \bar{M}\right) .
$$

Since $F_{d} \bar{M}=0$ for $d<0$, we have

$$
F_{d}\left(\Omega_{A / R}^{n} \bigotimes_{A} \bar{M}\right)=0 \quad \text { for } d<N-n .
$$


Note that by (4.6),

$$
F_{0} \bar{M}=F_{0} M_{C} / \sum_{j=1}^{r} D_{y_{j}}\left(F_{0} M_{C}\right) .
$$

Identifying $F_{0} M_{C}$ with $M$ and using $D_{y_{j}}(M)=f_{j} M$, we get

$$
F_{0} \bar{M}=M / \sum_{j=1}^{r} f_{j} M \simeq M_{B}
$$

Thus for all $n$ we have the identification

$$
F_{N-n}\left(\Omega_{A / R}^{n} \bigotimes_{A} \bar{M}\right)=\Omega_{A / R}^{n} \bigotimes_{A} M_{B}
$$

We define a map $\Phi: \Omega_{A / R}^{n} \bigotimes_{A} M_{B} \rightarrow \Omega_{A / R}^{n+r} \bigotimes_{A} M_{B}$ by the formula

$$
\Phi(\xi)=(-1)^{n r} d_{A / R} f_{1} \wedge \cdots \wedge d_{A / R} f_{r} \wedge \xi,
$$

where the exterior product on the right-hand side denotes the image of

$$
\left(d_{A / R} f_{1} \wedge \cdots \wedge d_{A / R} f_{r}\right) \otimes \xi \in \Omega_{A / R}^{r} \bigotimes_{A}\left(\Omega_{A / R}^{n} \bigotimes_{A} M_{B}\right)
$$

in $\Omega_{A / R}^{n+r} \bigotimes_{A} M_{B}$ under the canonical map.

Lemma 4.9 $\operatorname{ker} \Phi=\sum_{j=1}^{r}\left(\Omega_{A / R}^{n-1} \wedge d_{A / R} f_{j}\right) \bigotimes_{A} M_{B}$

Proof. It suffices to check equality locally. Since $M$ is a projective $A$-module we may assume $M$ is free and thus reduce to the case $M=A$. Localizing further if necessary, we get that $\Omega_{A / R}^{1} \otimes_{A} B$ is a free $B$-module of rank $N$ and

$$
\Omega_{A / R}^{n} \bigotimes_{A} B \simeq \bigwedge^{n}\left(\Omega_{A / R}^{1} \bigotimes_{A} B\right)
$$

(isomorphism of $B$-modules). We are thus in the situation of [19]. The smooth complete intersection hypothesis implies that the ideal of $B$ denoted by the symbol "script- $A$ " in [19] is the unit ideal. The desired conclusion then follows from part (i) of the theorem of [19].

Using the identification

$$
\Omega_{A / R}^{n} \bigotimes_{A} M_{B} /\left(\sum_{j=1}^{r}\left(\Omega_{A / R}^{n-1} \wedge d_{A / R} f_{j}\right) \bigotimes_{A} M_{B}\right) \simeq \Omega_{B / R}^{n} \bigotimes_{B} M_{B}
$$

and the identification (4.7), we see that, by the lemma, $\Phi$ induces an imbedding

$$
\bar{\Phi}: \Omega_{B / R}^{n} \bigotimes_{B} M_{B} \hookrightarrow \Omega_{A / R}^{n+r} \bigotimes_{A} \bar{M} \quad \text { for all } n .
$$

We show that $\bar{\Phi}$ is a homomorphism of complexes. Every element of $\Omega_{B / R}^{n} \bigotimes_{B} M_{B}$ is a sum of elements of the form $\bar{\omega} \otimes \bar{m}$ with $\omega \in \Omega_{A / R}^{n}$ and $m \in M$, where $\bar{\omega}$ denotes the image of $\omega$ under the composition

$$
\Omega_{A / R}^{n} \simeq \Omega_{A / R}^{n} \bigotimes_{A} A \rightarrow \Omega_{A / R}^{n} \bigotimes_{A} B \rightarrow \Omega_{B / R}^{n}
$$

and $\bar{m}$ is the image of $m$ under the canonical surjection $M \rightarrow M_{B}$. Thus to show $\bar{\Phi}$ is a homomorphism of complexes, it suffices to show

$$
\delta(\bar{\Phi}(\bar{\omega} \otimes \bar{m}))=\bar{\Phi}\left(\nabla_{B}(\bar{\omega} \otimes \bar{m})\right) .
$$


If $\nabla(m)$ is given by (4.2), then the definition of $\nabla_{B}$ gives

$$
\nabla_{B}(\bar{m})=\sum_{k} \bar{\omega}_{k} \otimes \bar{m}_{k}
$$

hence by (2.4) we have

$$
\nabla_{B}(\bar{\omega} \otimes \bar{m})=d_{B / R} \bar{\omega} \otimes \bar{m}+(-1)^{n} \sum_{k}\left(\bar{\omega} \wedge \bar{\omega}_{k}\right) \otimes \bar{m}_{k}
$$

This gives

$$
\begin{aligned}
\bar{\Phi}\left(\nabla_{B}(\bar{\omega} \otimes \bar{m})\right)= & (-1)^{n r+r}\left(d_{A / R} f_{1} \wedge \cdots \wedge d_{A / R} f_{r} \wedge d_{A / R} \omega\right) \otimes \bar{m}+ \\
& (-1)^{n r+r+n} \sum_{k}\left(d_{A / R} f_{1} \wedge \cdots \wedge d_{A / R} f_{r} \wedge \omega \wedge \omega_{k}\right) \otimes \bar{m}_{k} .
\end{aligned}
$$

From the definition of $\bar{\Phi}$ we have

$$
\bar{\Phi}(\bar{\omega} \otimes \bar{m})=(-1)^{n r}\left(d_{A / R} f_{1} \wedge \cdots \wedge d_{A / R} f_{r} \wedge \omega\right) \otimes[m],
$$

hence from $(4.4)$

$$
\begin{aligned}
\delta(\bar{\Phi}(\bar{\omega} \otimes \bar{m}))= & (-1)^{n r} d_{A / R}\left(d_{A / R} f_{1} \wedge \cdots \wedge d_{A / R} f_{r} \wedge \omega\right) \otimes[m]+ \\
& (-1)^{n r+n+r} d_{A / R} f_{1} \wedge \cdots \wedge d_{A / R} f_{r} \wedge \omega \wedge \delta([m]) \\
= & (-1)^{n r+r}\left(d_{A / R} f_{1} \wedge \cdots \wedge d_{A / R} f_{r} \wedge d_{A / R} \omega\right) \otimes[m]+ \\
& (-1)^{n r+n+r} \sum_{k}\left(d_{A / R} f_{1} \wedge \cdots \wedge d_{A / R} f_{r} \wedge \omega \wedge \omega_{k}\right) \otimes\left[m_{k}\right] .
\end{aligned}
$$

Under the identification (4.7) we have $\left[m_{k}\right]=\bar{m}_{k}$, which completes the proof of (4.10).

We now check that the map

$$
H_{\mathrm{DR}}^{n}\left(B / R,\left(M_{B}, \nabla_{B}\right)\right) \rightarrow H_{\mathrm{DR}}^{n+r}(A / R,(\bar{M}, \delta))
$$

induced by $\bar{\Phi}$ on cohomology respects the Gauss-Manin connection. We first observe that in the definition of $\bar{\Phi}$, we can replace $R$ by $\mathbf{C}$. The map

$$
\Phi_{\mathbf{C}}: \Omega_{A / \mathbf{C}}^{n} \bigotimes_{A} M_{B} \rightarrow \Omega_{A / \mathbf{C}}^{n+r} \bigotimes_{A} M_{B}
$$

defined by

$$
\Phi_{\mathbf{C}}(\xi)=d_{A / \mathbf{C}} f_{1} \wedge \cdots \wedge d_{A / \mathbf{C}} f_{r} \wedge \xi
$$

induces a homomorphism of complexes

$$
\bar{\Phi}_{\mathbf{C}}: \Omega_{B / \mathbf{C}} \bigotimes_{B} M_{B} \rightarrow \Omega_{A / \mathbf{C}}^{\cdot}[-r] \bigotimes_{A} \bar{M} .
$$

The Gauss-Manin connection is obtained from the $E_{1}$-terms of a spectral sequence associated to a certain filtration on the de Rham complex over $\mathbf{C}$. To check that $\bar{\Phi}$ respects the Gauss-Manin connection, it suffices to show that $\bar{\Phi}_{\mathbf{C}}$ respects this filtration, i. e., is a homomorphism of filtered complexes.

These (decreasing) filtrations, which we denote by $G$, are given by

$$
\begin{aligned}
G^{i} \Omega_{B / \mathbf{C}} & =\text { image of }\left(\Omega_{R / \mathbf{C}}^{i} \bigotimes_{R} B\right) \bigotimes_{B} \Omega_{B / \mathbf{C}}^{-i} \rightarrow \Omega_{B / \mathbf{C}}^{\cdot} \\
G^{i} \Omega_{A / \mathbf{C}} & =\text { image of }\left(\Omega_{R / \mathbf{C}}^{i} \bigotimes_{R} A\right) \bigotimes_{A} \Omega_{A / \mathbf{C}}^{\cdot-i} \rightarrow \Omega_{A / \mathbf{C}} .
\end{aligned}
$$


The surjection $\Omega_{A / \mathbf{C}}^{i} \rightarrow \Omega_{B / \mathbf{C}}^{i}$ induces the surjection $\Omega_{R / \mathbf{C}}^{i} \bigotimes_{R} A \rightarrow \Omega_{R / \mathbf{C}}^{i} \bigotimes_{R} B$. Thus if $\bar{\omega} \in G^{i} \Omega_{B / \mathrm{C}}^{n}$, then we can choose a lifting $\omega \in G^{i} \Omega_{A / \mathbf{C}}^{n}$. This filtration is compatible with exterior product, hence

$$
d_{A / \mathbf{C}} f_{1} \wedge \cdots \wedge d_{A / \mathbf{C}} f_{r} \wedge \omega \in G^{i} \Omega_{A / \mathbf{C}}^{n+r}
$$

It follows from (4.11) that $\bar{\Phi}_{\mathbf{C}}\left(G^{i} \Omega_{B / \mathbf{C}} \bigotimes_{B} M_{B}\right) \subseteq G^{i} \Omega_{A / \mathbf{C}}[-r] \bigotimes_{A} \bar{M}$.

To complete this section, we determine the image of $\bar{\Phi}$. We define a subcomplex $L^{\cdot}$ of $\Omega_{A / R}^{\cdot} \otimes_{A} \bar{M}$ by setting

$$
L^{n}=\left\{\xi \in F_{N-n}\left(\Omega_{A / R}^{n} \bigotimes_{A} \bar{M}\right) \mid \delta(\xi) \in F_{N-n-1}\left(\Omega_{A / R}^{n+1} \bigotimes_{A} \bar{M}\right)\right\}
$$

Proposition 4.12 Suppose $f_{1}, \ldots, f_{r}$ define a smooth complete intersection $Y \subseteq X$ and $M$ is a projective $A$-module of finite rank. Then $\bar{\Phi}$ is an isomorphism of complexes from $\Omega_{B / R} \otimes_{B} M_{B}$ onto $L \cdot[-r]$.

We begin with a lemma.

Lemma 4.13 Suppose $\omega \in \Omega_{A / R}^{n+r} \bigotimes_{A} M_{B}$ satisfies

$$
d_{A / R} f_{j} \wedge \omega=0 \quad \text { for } j=1, \ldots, r .
$$

Then $\omega \in \operatorname{im} \Phi$.

Proof. It suffices to check the condition locally, i. e., to show that for any maximal ideal $\mathbf{p}$ of $A$, if

$$
\left(d_{A / R} f_{j}\right)_{\mathbf{p}} \wedge \omega_{\mathbf{p}}=0 \quad \text { for } j=1, \ldots, r,
$$

then $\omega_{\mathbf{p}} \in \operatorname{im} \Phi_{\mathbf{p}}$. Since $\Omega_{A / R} \bigotimes_{A} M_{B}$ is supported on $Y$, we may assume $\mathbf{p}$ corresponds to a point of $Y$. Furthermore, since $M$ is projective, we may assume that $M$ is a free $A$-module and thus reduce to the case $M=A$. But the smooth complete intersection hypothesis implies that $\left(d_{A / R} f_{1}\right)_{\mathbf{p}}, \ldots,\left(d_{A / R} f_{r}\right)_{\mathbf{p}}$ can be extended to a basis of $\left(\Omega_{A / R}^{1}\right)_{\mathbf{p}}$ as $A_{\mathbf{p}}$-module and $\left(d_{A / R} f_{1}\right)_{\mathbf{p}} \otimes 1, \ldots,\left(d_{A / R} f_{r}\right)_{\mathbf{p}} \otimes 1$ can be extended to a basis of $\left(\Omega_{A / R}^{1}\right)_{\mathbf{p}} \bigotimes_{A_{\mathbf{p}}} B_{\mathbf{p}^{\prime}}$ as $B_{\mathbf{p}^{\prime}}$-module, where $\mathbf{p}^{\prime}$ denotes the image of $\mathbf{p}$ in $B$. Since

$$
\left(\Omega_{A / R}^{n}\right)_{\mathbf{p}} \bigotimes_{A_{\mathbf{p}}} B_{\mathbf{p}}=\bigwedge^{n}\left(\left(\Omega_{A / R}^{1}\right)_{\mathbf{p}} \bigotimes_{A_{\mathbf{p}}} B_{\mathbf{p}}\right)
$$

the result follows immediately.

Proof of Proposition 4.12. We have already proved that $\bar{\Phi}$ is an injective homomorphism of complexes and (4.10) shows that its image is contained in $L^{\cdot}[-r]$. So it only remains to prove $L^{*}[-r] \subseteq \operatorname{im} \bar{\Phi}$. Suppose $\xi \in F_{N-n}\left(\Omega_{A / R}^{n} \otimes_{A} \bar{M}\right)$. We may write

$$
\xi=\sum_{k} \omega_{k} \otimes\left[m_{k}\right]
$$

where $\omega_{k} \in \Omega_{A / R}^{n}$ and $m_{k} \in M$. Write

$$
\nabla\left(m_{k}\right)=\sum_{l} \omega_{k l} \otimes m_{k l}
$$

with $\omega_{k l} \in \Omega_{A / R}^{1}$ and $m_{k l} \in M$. By (4.4) we have

$$
\delta(\xi)=\sum_{k} d_{A / R} \omega_{k} \otimes\left[m_{k}\right]+(-1)^{n} \sum_{k} \sum_{l}\left(\omega_{k} \wedge \omega_{k l}\right) \otimes\left[m_{k l}\right]+(-1)^{n} \sum_{j=1}^{r} \sum_{k}\left(d_{A / R} f_{j} \wedge \omega_{k}\right) \otimes\left[y_{j} m_{k}\right] .
$$

Using (4.1) and (3.5), we see that $\delta(\xi) \in F_{N-n-1}\left(\Omega_{A / R}^{n+1} \bigotimes_{A} \bar{M}\right)$ (and hence $\left.\xi \in L^{n}\right)$ if and only if

$$
d_{A / R} f_{j} \wedge\left(\sum_{k} \omega_{k} \otimes\left[m_{k}\right]\right)=0 \quad \text { for } j=1, \ldots, r .
$$

By Lemma 4.13, this implies $\xi \in \operatorname{im} \bar{\Phi}$. 


\section{$\S 5 . \quad$ Completion of the proof}

To complete the proof of Theorem 4.5, it suffices by Proposition 4.12 to show the following.

Theorem 5.1 Suppose $f_{1}, \ldots, f_{r}$ define a smooth complete intersection $Y \subseteq X$ and $M$ is a projective $A$ module of finite rank. Then the inclusion $L^{\cdot} \hookrightarrow \Omega_{A / R}^{\cdot} \otimes_{A} \bar{M}$ is a quasi-isomorphism.

We begin with a lemma. Let $T$ be a local $R$-algebra that is smooth over $R$ of relative dimension $N$ and let $f_{1}, \ldots, f_{N}$ be elements of the maximal ideal of $T$ such that $d_{T / R} f_{1}, \ldots, d_{T / R} f_{N}$ form a basis for $\Omega_{T / R}^{1}$. Let $T^{\prime}$ be a $T$-algebra, let $y_{1}, \ldots, y_{r}$ be indeterminates, and consider

$$
\Omega_{T / R}^{n} \bigotimes_{T} T^{\prime}\left[y_{1}, \ldots, y_{r}\right]
$$

It is a free $T^{\prime}\left[y_{1}, \ldots, y_{r}\right]$-module with basis

$$
\left\{d_{T / R} f_{i_{1}} \wedge \cdots \wedge d_{T / R} f_{i_{n}} \mid 1 \leq i_{1}<\cdots<i_{n} \leq N\right\}
$$

and is a free $T^{\prime}$-module with basis

$$
\left\{y_{1}^{a_{1}} \cdots y_{r}^{a_{r}} d_{T / R} f_{i_{1}} \wedge \cdots \wedge d_{T / R} f_{i_{n}} \mid a_{1}, \ldots, a_{r} \in \mathbf{N}, 1 \leq i_{1}<\cdots<i_{n} \leq N\right\} .
$$

We grade $T^{\prime}\left[y_{1}, \ldots, y_{r}\right]$ by degee, i. e.,

$$
T^{\prime}\left[y_{1}, \ldots, y_{r}\right]^{(d)}=T^{\prime} \text {-span of } y_{1}^{a_{1}} \cdots y_{r}^{a_{r}} \text { with } a_{1}+\cdots+a_{r}=d
$$

and we grade $\Omega_{T / R}^{n} \bigotimes_{T} T^{\prime}\left[y_{1}, \ldots, y_{r}\right]$ by

$$
\operatorname{gr}^{(d)}\left(\Omega_{T / R}^{n} \bigotimes_{T} T^{\prime}\left[y_{1}, \ldots, y_{r}\right]\right)=\Omega_{T / R}^{n} \bigotimes_{T} T^{\prime}\left[y_{1}, \ldots, y_{r}\right]^{(d+n-N)} .
$$

The map $\Omega_{T / R}^{n} \bigotimes_{T} T^{\prime}\left[y_{1}, \ldots, y_{r}\right] \rightarrow \Omega_{T / R}^{n+1} \bigotimes_{T} T^{\prime}\left[y_{1}, \ldots, y_{r}\right]$ defined by

$$
\omega \mapsto \sum_{j=1}^{r} y_{j} d_{T / R} f_{j} \wedge \omega
$$

then makes $\Omega_{T / R} \otimes_{T} T^{\prime}\left[y_{1}, \ldots, y_{r}\right]$ into a graded complex of $T^{\prime}$-modules.

Lemma 5.5 With notation and hypotheses as above, we have

$$
H^{n}\left(\operatorname{gr}^{(d)}\left(\Omega_{T / R} \bigotimes_{T} T^{\prime}\left[y_{1}, \ldots, y_{r}\right]\right)\right)=0 \quad \text { for } d>N-n .
$$

Proof. The proof is by induction on $r$. Suppose $r=1$ and let

$$
\omega \in \Omega_{T / R}^{n} \bigotimes_{T} T^{\prime}\left[y_{1}\right]^{(d+n-N)} .
$$

The condition $d>N-n$ implies that $\omega$ is divisible by $y_{1}$, i. e., $\omega$ can be written

$$
\omega=\sum_{1 \leq i_{1}<\cdots<i_{n} \leq N} y_{1} \omega\left(i_{1}, \ldots, i_{n}\right) d_{T / R} f_{i_{1}} \wedge \cdots \wedge d_{T / R} f_{i_{n}}
$$

with $\omega\left(i_{1}, \ldots, i_{n}\right) \in T^{\prime}\left[y_{1}\right]^{(d+n-N-1)}$. The condition that $\omega$ be a cocycle is that

$$
d_{T / R} f_{1} \wedge \omega=0
$$


Since (5.2) is a basis, we see that this is the case if and only if $\omega\left(i_{1}, \ldots, i_{n}\right) \neq 0$ implies $i_{1}=1$. Put

$$
\xi=\sum_{2 \leq i_{2}<\cdots<i_{n} \leq N} \omega\left(1, i_{2}, \ldots, i_{n}\right) d_{T / R} f_{i_{2}} \wedge \cdots \wedge d_{T / R} f_{i_{n}} .
$$

Then $\xi \in \operatorname{gr}^{(d)}\left(\Omega_{T / R}^{n-1} \bigotimes_{T} T^{\prime}\left[y_{1}\right]\right)$ and

$$
\omega=y_{1} d_{T / R} f_{1} \wedge \xi
$$

so $\omega$ is a coboundary.

Now suppose the lemma true for $r-1$ and let

$$
\omega \in \operatorname{gr}^{(d)}\left(\Omega_{T / R}^{n} \bigotimes_{T} T^{\prime}\left[y_{1}, \ldots, y_{r}\right]\right) .
$$

Let $h$ be the highest power of $y_{1}$ appearing in any term of $\omega$ (in the decomposition corresponding to the basis (5.3)) and let $\omega^{(h)}$ be the sum of all terms of $\omega$ of degree $h$ in $y_{1}$. Suppose $h>0$. Looking at the terms of degree $h+1$ in $y_{1}$ in the cocycle equation

$$
\sum_{j=1}^{r} y_{j} d_{T / R} f_{j} \wedge \omega=0
$$

gives

$$
d_{T / R} f_{1} \wedge \omega^{(h)}=0
$$

hence

$$
\omega^{(h)}=\sum_{2 \leq i_{2}<\cdots<i_{n} \leq N} y_{1} \xi\left(i_{2}, \ldots, i_{n}\right) d_{T / R} f_{1} \wedge d_{T / R} f_{i_{2}} \wedge \cdots \wedge d_{T / R} f_{i_{n}}
$$

for some $\xi\left(i_{2}, \ldots, i_{n}\right) \in T^{\prime}\left[y_{1}, \ldots, y_{r}\right]^{(d+n-N-1)}$. Put

$$
\xi=\sum_{2 \leq i_{2}<\cdots<i_{n} \leq N} \xi\left(i_{2}, \ldots, i_{n}\right) d_{T / R} f_{i_{2}} \wedge \cdots \wedge d_{T / R} f_{i_{n}} .
$$

Then $\xi \in \operatorname{gr}^{(d)}\left(\Omega_{T / R}^{n-1} \otimes_{T} T^{\prime}\left[y_{1}, \ldots, y_{r}\right]\right)$ and the highest power of $y_{1}$ appearing in $\omega-\left(\sum_{j=1}^{r} y_{j} d_{T / R} f_{j} \wedge \xi\right)$ is $\leq h-1$.

We may thus reduce to the case $h=0$, i. e., $y_{1}$ does not appear in $\omega$. The cocycle equation (5.6) then implies

$$
d_{T / R} f_{1} \wedge \omega=0
$$

and

$$
\sum_{j=2}^{r} y_{j} d_{T / R} f_{j} \wedge \omega=0 .
$$

From (5.7) we have

$$
\omega=\sum_{2 \leq i_{2}<\cdots<i_{n} \leq N} \omega\left(i_{2}, \ldots, i_{n}\right) d_{T / R} f_{1} \wedge d_{T / R} f_{i_{2}} \wedge \cdots \wedge d_{T / R} f_{i_{n}}
$$

with $\omega\left(i_{2}, \ldots, i_{n}\right) \in T^{\prime}\left[y_{2}, \ldots, y_{r}\right]^{(d+n-N)}$. Put

$$
\omega^{\prime}=\sum_{2 \leq i_{2}<\cdots<i_{n} \leq N} \omega\left(i_{2}, \ldots, i_{n}\right) d_{T / R} f_{i_{2}} \wedge \cdots \wedge d_{T / R} f_{i_{n}} \in \operatorname{gr}^{(d+1)}\left(\Omega_{T / R}^{n-1} \bigotimes_{T} T^{\prime}\left[y_{2}, \ldots, y_{r}\right]\right) .
$$

From (5.8) we have

$$
\sum_{j=2}^{r} y_{j} d_{T / R} f_{j} \wedge \omega^{\prime}=0
$$


so by the induction hypothesis there exists

$$
\xi^{\prime} \in \operatorname{gr}^{(d+1)}\left(\Omega_{T / R}^{n-2} \bigotimes_{T} T^{\prime}\left[y_{2}, \ldots, y_{r}\right]\right)
$$

such that

$$
\sum_{j=2}^{r} y_{j} d_{T / R} f_{j} \wedge \xi^{\prime}=\omega^{\prime}
$$

If we put

$$
\xi=-d_{T / R} f_{1} \wedge \xi^{\prime} \in \operatorname{gr}^{(d)}\left(\Omega_{T / R}^{n-1} \bigotimes_{T} T^{\prime}\left[y_{1}, \ldots, y_{r}\right]\right)
$$

then (5.9) implies

$$
\begin{aligned}
\sum_{j=1}^{r} y_{j} d_{T / R} f_{j} \wedge \xi & =d_{T / R} f_{1} \wedge \omega^{\prime} \\
& =\omega,
\end{aligned}
$$

hence $\omega$ is a coboundary. This completes the proof of Lemma 5.5.

Proof of Theorem 5.1. To show that the inclusion $L^{\cdot} \hookrightarrow \Omega_{A / R}^{\cdot} \otimes_{A} \bar{M}$ is a quasi-isomorphism, it suffices to show that the corresponding map of associated graded complexes (relative to the filtration $F$. defined previously)

$$
\operatorname{gr}^{F}\left(L^{*}\right) \hookrightarrow \operatorname{gr}^{F}\left(\Omega_{A / R}^{\cdot} \bigotimes_{A} \bar{M}\right)
$$

is a quasi-isomorphism. But $F$. induces the "stupid" filtration on $L$ :

$$
F_{d} L^{n}= \begin{cases}L^{n} & \text { if } d \geq N-n, \\ 0 & \text { if } d<N-n,\end{cases}
$$

hence $\operatorname{gr}_{d}^{F}\left(L^{*}\right)$ is the complex with $L^{N-d}$ in degree $N-d$ and zeros elsewhere if $0 \leq d \leq N$ and is the zero complex otherwise. Thus the assertion that (5.10) is a quasi-isomorphism is equivalent to the assertion that

$$
0 \rightarrow L^{N-d} \rightarrow F_{d}\left(\Omega_{A / R}^{N-d} \bigotimes_{A} \bar{M}\right) \rightarrow \operatorname{gr}_{d}^{F}\left(\Omega_{A / R}^{N+1-d} \bigotimes_{A} \bar{M}\right) \rightarrow \cdots \rightarrow \operatorname{gr}_{d}^{F}\left(\Omega_{A / R}^{N} \bigotimes_{A} \bar{M}\right) \rightarrow 0
$$

is exact for $0 \leq d \leq N$ and

$$
0 \rightarrow \operatorname{gr}_{d}^{F}\left(\Omega_{A / R}^{0} \bigotimes_{A} \bar{M}\right) \rightarrow \cdots \rightarrow \operatorname{gr}_{d}^{F}\left(\Omega_{A / R}^{N} \bigotimes_{A} \bar{M}\right) \rightarrow 0
$$

is exact for $d>N$. The definition of $L^{\cdot}$ shows that the sequence

$$
0 \rightarrow L^{N-d} \rightarrow F_{d}\left(\Omega_{A / R}^{N-d} \bigotimes_{A} \bar{M}\right) \rightarrow \operatorname{gr}_{d}^{F}\left(\Omega_{A / R}^{N+1-d} \bigotimes_{A} \bar{M}\right)
$$

is exact for $0 \leq d \leq N$. Thus we need to show that the sequence

$$
\operatorname{gr}_{d}^{F}\left(\Omega_{A / R}^{n-1} \bigotimes_{A} \bar{M}\right) \rightarrow \operatorname{gr}_{d}^{F}\left(\Omega_{A / R}^{n} \bigotimes_{A} \bar{M}\right) \rightarrow \operatorname{gr}_{d}^{F}\left(\Omega_{A / R}^{n+1} \bigotimes_{A} \bar{M}\right)
$$

is exact whenever $d>N-n$, i. e., that

$$
H^{n}\left(\operatorname{gr}_{d}^{F}\left(\Omega_{A / R} \bigotimes_{A} \bar{M}\right)\right)=0 \quad \text { whenever } d>N-n
$$


There are natural identifications of $A$-modules

$$
\begin{aligned}
\operatorname{gr}_{d}^{F}\left(\Omega_{A / R}^{n} \bigotimes_{A} \bar{M}\right) & \simeq \Omega_{A / R}^{n} \bigotimes_{A}\left(F_{d+n-N} M_{C} /\left(F_{d+n-N-1} M_{C}+\sum_{j=1}^{r} D_{y_{j}}\left(F_{d+n-N} M_{C}\right)\right)\right) \\
& \simeq \Omega_{A / R}^{n} \bigotimes_{A}\left(M_{C}^{(d+n-N)} / \sum_{j=1}^{r} f_{j} M_{C}^{(d+n-N)}\right) \\
& \simeq \Omega_{A / R}^{n} \bigotimes_{A}\left(M \bigotimes_{A} B\left[y_{1}, \ldots, y_{r}\right]^{(d+n-N)}\right) .
\end{aligned}
$$

We are thus reduced to proving

$$
H^{n}\left(\operatorname{gr}^{(d)}\left(\Omega_{A / R}^{n} \bigotimes_{A}\left(M \bigotimes_{A} B\left[y_{1}, \ldots, y_{r}\right]\right)\right)\right)=0 \quad \text { for } d>N-n
$$

where

$$
\operatorname{gr}^{(d)}\left(\Omega_{A / R}^{n} \bigotimes_{A}\left(M \bigotimes_{A} B\left[y_{1}, \ldots, y_{r}\right]\right)\right)=\Omega_{A / R}^{n} \bigotimes_{A}\left(M \bigotimes_{A} B\left[y_{1}, \ldots, y_{r}\right]^{(d+n-N)}\right)
$$

and the coboundary map of the complex is given by

$$
\omega \mapsto \sum_{j=1}^{r} y_{j} d_{A / R} f_{j} \wedge \omega .
$$

To prove the vanishing result (5.12), we may first localize at a maximal ideal $\mathbf{p}$ of $A$. Since $M$ is a projective $A$-module, we may assume $M$ is free and thus reduce to the case $M=A$. Furthermore, this complex is supported on $Y$, so we may suppose $\mathbf{p}$ corresponds to a point of $Y$. We thus want to show that

$$
H^{n}\left(\operatorname{gr}^{(d)}\left(\left(\Omega_{A / R}^{n}\right)_{\mathbf{p}} \bigotimes_{A_{\mathbf{p}}} B_{\mathbf{p}^{\prime}}\left[y_{1}, \ldots, y_{r}\right]\right)\right)=0 \quad \text { for } d>N-n,
$$

where $\mathbf{p}^{\prime}$ denotes the image of $\mathbf{p}$ in $B$. But the smooth complete intersection hypothesis implies that there exist $f_{r+1}, \ldots, f_{N} \in A_{\mathbf{p}}$ such that $d_{A_{\mathbf{p}} / R} f_{1}, \ldots, d_{A_{\mathbf{p}} / R} f_{N}$ form a basis for $\Omega_{A_{\mathbf{p}} / R}^{1} \simeq\left(\Omega_{A / R}^{1}\right)_{\mathbf{p}}$. The result then follows by applying Lemma 5.5 with $T=A_{\mathbf{p}}$ and $T^{\prime}=B_{\mathbf{p}^{\prime}}$.

\section{$\S 6 . \quad$ Hypergeometric equations}

In this section we fix $X=\mathbf{A}_{R}^{N}$ and discuss the case of a smooth complete intersection $Y \subseteq \mathbf{A}_{R}^{N}$ defined by $f_{1}, \ldots, f_{r} \in R\left[x_{1}, \ldots, x_{N}\right]$. In the notation of the previous sections, we have

$$
\begin{aligned}
& A=R\left[x_{1}, \ldots, x_{N}\right] \\
& B=R\left[x_{1}, \ldots, x_{N}\right] /\left(f_{1}, \ldots, f_{r}\right) \\
& C=R\left[x_{1}, \ldots, x_{N}, y_{1}, \ldots, y_{r}\right] .
\end{aligned}
$$

We take $M=A$ with the standard connection, hence $M_{B}=B$ with the standard connection and we are computing the usual de Rham cohomology of the variety $Y$. Theorem 2.5 gives (where $S=\operatorname{Spec}(R)$ )

$$
H_{\mathrm{DR}}^{n}(Y / S) \simeq H_{\mathrm{DR}}^{n+2 r}\left(C / R,\left(C, \nabla_{F}\right)\right),
$$

where $\nabla_{F}$ is the connection

$$
\nabla_{F}(\mu)=d_{C / R} \mu+d_{C / R} F \otimes \mu
$$

with $\mu \in C, F=y_{1} f_{1}+\cdots+y_{r} f_{r} \in C$. Let $D_{R}$ be the ring of $\mathbf{C}$-linear differential operators with coefficients in $R$, i. e., $D_{R}$ is the subring of $\operatorname{End}_{\mathbf{C}}(R)$ generated by $R$ and $\operatorname{Der}_{\mathbf{C}}(R)$.

We will be mainly interested in $H_{\mathrm{DR}}^{N-r}(Y / R)$. As noted in the introduction, this is given by (1.2) and the action of a derivation $\partial \in \operatorname{Der}_{\mathbf{C}}(R)$ on $H_{\mathrm{DR}}^{N-r}(Y / S)$ is given by (1.5). We shall see that the differential 
equations satisfied by cohomology classes of $(N-r)$-forms on $Y$ can be determined from the monomials that appear in the $f_{j}$. For $j=1, \ldots, r$, write

$$
f_{j}=\sum_{i=1}^{\delta_{j}} \lambda_{j, i} x^{d_{j, i}},
$$

where $\lambda_{j, i} \in R$ and each $d_{j, i}$ is an $N$-tuple of nonnegative integers. Let

$$
\begin{aligned}
F & =\sum_{j=1}^{r} y_{j} f_{j} \\
& =\sum_{j=1}^{r} \sum_{i=1}^{\delta_{j}} \lambda_{j, i} x^{d_{j, i}} y^{e_{j}},
\end{aligned}
$$

where $e_{1}, \ldots, e_{r}$ are the standard unit basis vectors in $\mathbf{R}^{r}$. We let $\left[x^{u} y^{v}\right] \in H_{\mathrm{DR}}^{N+r}\left(C / R,\left(C, \nabla_{F}\right)\right)$ be the cohomology class represented by the differential form

$$
x^{u} y^{v} d x_{1} \wedge \cdots \wedge d x_{N} \wedge d y_{1} \wedge \cdots \wedge d y_{r} \in \Omega_{C / R}^{N+r} .
$$

We define for each $u \in \mathbf{N}^{N}$ an $(N-r)$-form $\omega_{u} \in \Omega_{B / R}^{N-r}$ as follows. For any subset $\sigma=\left\{i_{1}, \ldots, i_{r}\right\} \subseteq$ $\{1, \ldots, N\}$, put

$$
J_{\sigma}=\operatorname{det}\left[\frac{\partial f_{j}}{\partial x_{i_{k}}}\right]_{j, k=1, \ldots, r}
$$

and let $Y_{\sigma} \subseteq Y$ be the open subset where $J_{\sigma}$ is invertible. Consider the differential $(N-r)$-form on $Y_{\sigma}$

$$
\frac{(\operatorname{sgn} \sigma) x^{u}}{J_{\sigma}} d x_{1} \wedge \cdots \wedge \widehat{d x_{i_{1}}} \wedge \cdots \wedge \widehat{d x_{i_{r}}} \wedge \cdots \wedge d x_{N}
$$

where $\operatorname{sgn} \sigma= \pm 1$ is chosen so that

$$
(\operatorname{sgn} \sigma) d x_{1} \wedge \cdots \wedge \widehat{d x_{i_{1}}} \wedge \cdots \wedge \widehat{d x_{i_{r}}} \wedge \cdots \wedge d x_{N} \wedge d x_{i_{1}} \wedge \cdots \wedge d x_{i_{r}}=d x_{1} \wedge \cdots \wedge d x_{N} .
$$

One checks that these forms agree on overlaps $Y_{\sigma} \cap Y_{\sigma^{\prime}}$, hence define a global $(N-r)$-form $\omega_{u} \in \Omega_{B / R}^{N-r}$. Let $\left[\omega_{u}\right]$ denote the cohomology class of $\omega_{u}$ in $H_{\mathrm{DR}}^{N-r}(Y / S)$. One checks that $\left[\omega_{u}\right]$ corresponds to $\left[x^{u}\right]$ under the isomorphism of Theorem 2.5. Note that by Theorem 5.1, the cohomology classes $\left[x^{u}\right]$ span $H_{\mathrm{DR}}^{N+r}\left(C / R,\left(C, \nabla_{F}\right)\right)$, hence by Theorem 2.5 the classes $\left[\omega_{u}\right]$ span $H_{\mathrm{DR}}^{N-r}(Y / S)$.

The main purpose of this section is to describe a left ideal $I_{R}(u, v)$ of $D_{R}$ that annihilates $\left[x^{u} y^{v}\right]$. By the preceding remarks, $I_{R}(u, 0)$ then annihilates the cohomology class $\left[\omega_{u}\right] \in H_{\mathrm{DR}}^{N-r}(Y / S)$. To define these left ideals, we define them in the "generic" case, and take the "pullback" to $Y$.

By the "generic" case, we mean the following. Let $\left\{\mu_{j, i}\right\}\left(j=1, \ldots, r, i=1, \ldots, \delta_{j}\right)$ be a collection of indeterminates and $\mathbf{C}[\mu]$ the polynomial ring in these indeterminates. For $j=1, \ldots, r$, put

$$
\begin{aligned}
f_{j}^{(\mu)} & =\sum_{i=1}^{\delta_{j}} \mu_{j, i} x^{d_{j, i}}, \\
F^{(\mu)} & =\sum_{j=1}^{r} y_{j} f_{j}^{(\mu)} \\
& =\sum_{j=1}^{r} \sum_{i=1}^{\delta_{j}} \mu_{j, i} x^{d_{j, i}} y^{e_{j}} .
\end{aligned}
$$

Let $\mathbf{A}_{\mathbf{C}[\mu]}^{N}$ denote affine $N$-space over $\mathbf{C}[\mu]$ with coordinate ring $A_{\mu}=\mathbf{C}[\mu]\left[x_{1}, \ldots, x_{N}\right]$ and put $C_{\mu}=$ $A_{\mu}\left[y_{1}, \ldots, y_{r}\right]$, the coordinate ring of $\mathbf{A}_{\mathbf{C}[\mu]}^{N+r}$. Let $\nabla_{F^{(\mu)}}$ be the connection on $C_{\mu}$ defined by

$$
\nabla_{F^{(\mu)}}(\omega)=d_{C_{\mu} / \mathbf{C}[\mu]} \omega+d_{C_{\mu} / \mathbf{C}[\mu]} F^{(\mu)} \wedge \omega .
$$


We let $\left[x^{u} y^{v}\right]_{\mu} \in H_{\mathrm{DR}}^{N+r}\left(C_{\mu} / \mathbf{C}[\mu],\left(C_{\mu}, \nabla_{F^{(\mu)}}\right)\right)$ be the cohomology class represented by the differential form

$$
x^{u} y^{v} d x_{1} \wedge \cdots \wedge d x_{N} \wedge d y_{1} \wedge \cdots \wedge d y_{r}
$$

Let $D_{\mathbf{C}[\mu]}$ be the ring of differential operators in the $\partial / \partial \mu_{j, i}$ with polynomial coefficients. We describe a collection of differential operators in $D_{\mathbf{C}[\mu]}$ that annihilate $\left[x^{u} y^{v}\right]_{\mu}$.

First we recall the definition of the hypergeometric system associated to the collection of lattice points $\left\{\left(d_{j, i}, e_{j}\right) \mid j=1, \ldots, r, i=1, \ldots, \delta_{j}\right\} \subseteq \mathbf{R}^{N+r}$ (see [11]). Let $E \subseteq \mathbf{Z}^{\delta_{1}+\cdots+\delta_{r}}$ be the group of relations among these lattice points, i. e.,

$$
E=\left\{\left(b_{j, i}\right) \mid \sum_{j=1}^{r} \sum_{i=1}^{\delta_{j}} b_{j, i}\left(d_{j, i}, e_{j}\right)=0\right\} .
$$

For $b=\left(b_{j, i}\right) \in E$, let $\square_{b}$ be the constant coefficient differential operator

$$
\square_{b}=\prod_{b_{j, i}>0}\left(\frac{\partial}{\partial \mu_{j, i}}\right)^{b_{j, i}}-\prod_{b_{j, i}<0}\left(\frac{\partial}{\partial \mu_{j, i}}\right)^{-b_{j, i}} .
$$

Write $d_{j, i}=\left(d_{j, i}(1), \ldots, d_{j, i}(N)\right) \in \mathbf{N}^{N}$. Define

$$
\begin{aligned}
Z_{k} & =\sum_{j=1}^{r} \sum_{i=1}^{\delta_{j}} d_{j, i}(k) \mu_{j, i} \frac{\partial}{\partial \mu_{j, i}} \quad \text { for } k=1, \ldots, N, \\
Z_{N+k} & =\sum_{i=1}^{\delta_{k}} \mu_{k, i} \frac{\partial}{\partial \mu_{k, i}} \quad \text { for } k=1, \ldots, r .
\end{aligned}
$$

Let $\beta=\left(\beta_{1}, \ldots, \beta_{N+r}\right) \in \mathbf{C}^{N+r}$. By the hypergeometric system with parameter $\beta$ associated to the collection of lattice points $\left\{\left(d_{j, i}, e_{j}\right)\right\}_{j, i}$ we mean the system of differential equations

$$
\begin{aligned}
& \square_{b}(Y)=0 \quad \text { for all } b \in E, \\
& Z_{k}(Y)=\beta_{k} Y \quad \text { for } k=1, \ldots, N+r .
\end{aligned}
$$

Recall([11], see also [1]) that this system is holonomic.

Theorem 6.4 The cohomology class $\left[x^{u} y^{v}\right]_{\mu} \in H_{\mathrm{DR}}^{N+r}\left(C_{\mu} / \mathbf{C}[\mu],\left(C_{\mu}, \nabla_{F^{(\mu)}}\right)\right)$ satisfies the hypergeometric system

$$
\begin{aligned}
& \square_{b}\left(\left[x^{u} y^{v}\right]_{\mu}\right)=0 \quad \text { for } b \in E \text {, } \\
& Z_{k}\left(\left[x^{u} y^{v}\right]_{\mu}\right)=-\left(u_{k}+1\right)\left[x^{u} y^{v}\right]_{\mu} \quad \text { for } k=1, \ldots, N, \\
& Z_{N+k}\left(\left[x^{u} y^{v}\right]_{\mu}\right)=-\left(v_{k}+1\right)\left[x^{u} y^{v}\right]_{\mu} \quad \text { for } k=1, \ldots, r \text {, }
\end{aligned}
$$

where we have written $u=\left(u_{1}, \ldots, u_{N}\right), v=\left(v_{1}, \ldots, v_{r}\right)$.

Proof. One computes directly from the definition of $\nabla_{F^{(\mu)}}$ that there is an isomorphism of $D_{\mathbf{C}[\mu]}$-modules

$$
H_{\mathrm{DR}}^{N+r}\left(C_{\mu} / \mathbf{C}[\mu],\left(C_{\mu}, \nabla_{F^{(\mu)}}\right)\right) \simeq C_{\mu} /\left(\sum_{i=1}^{N} D_{x_{i}}^{(\mu)}\left(C_{\mu}\right)+\sum_{j=1}^{r} D_{y_{j}}^{(\mu)}\left(C_{\mu}\right)\right),
$$

where

$$
\begin{aligned}
D_{x_{i}}^{(\mu)} & =\frac{\partial}{\partial x_{i}}+\sum_{j=1}^{r} y_{j} \frac{\partial f_{j}^{(\mu)}}{\partial x_{i}} \\
D_{y_{j}}^{(\mu)} & =\frac{\partial}{\partial y_{j}}+f_{j}^{(\mu)}
\end{aligned}
$$


Under this isomorphism, the cohomology class $\left[x^{u} y^{v}\right]_{\mu}$ in the left-hand side corresponds to the class of the monomial $x^{u} y^{v}$ in the quotient on the right-hand side. Furthermore, the action of $\partial / \partial \mu_{j, i}$ on $H_{\mathrm{DR}}^{N+r}\left(C_{\mu} / \mathbf{C}[\mu],\left(C_{\mu}, \nabla_{F^{(\mu)}}\right)\right)$ is induced by the action of the differential operator

$$
\begin{aligned}
D_{\mu_{j, i}} & =\frac{\partial}{\partial \mu_{j, i}}+\frac{\partial F^{(\mu)}}{\partial \mu_{j, i}} \\
& =\frac{\partial}{\partial \mu_{j, i}}+x^{d_{j, i}} y^{e_{j}}
\end{aligned}
$$

on $C_{\mu}$. In particular,

$$
\frac{\partial}{\partial \mu_{j, i}}\left(\left[x^{u} y^{v}\right]_{\mu}\right)=\left[x^{u+d_{j, i}} y^{v+e_{j}}\right]_{\mu} .
$$

More generally, if $\left\{b_{j, i}\right\}$ is a collection of nonnegative integers,

$$
\prod_{j, i}\left(\frac{\partial}{\partial \mu_{j, i}}\right)^{b_{j, i}}\left(\left[x^{u} y^{v}\right]_{\mu}\right)=\left[x^{u+\sum_{j, i} b_{j, i} d_{j, i}} y^{v+\sum_{j, i} b_{j, i} e_{j}}\right]_{\mu} .
$$

It follows immediately from the definition of $E$ that $\square_{b}\left(\left[x^{u} y^{v}\right]_{\mu}\right)=0$ for all $b \in E$.

Using (6.6), we see that for $k=1, \ldots, N$,

$$
\begin{aligned}
Z_{k}\left(\left[x^{u} y^{v}\right]_{\mu}\right) & =\left[\sum_{j=1}^{r} \sum_{i=1}^{\delta_{j}} d_{j, i}(k) \mu_{j, i} D_{\mu_{j, i}}\left(x^{u} y^{v}\right)\right]_{\mu} \\
& =\left[\sum_{j=1}^{r} \sum_{i=1}^{\delta_{j}} d_{j, i}(k) \mu_{j, i} x^{u+d_{j, i}} y^{v+e_{j}}\right]_{\mu} .
\end{aligned}
$$

Note that

$$
\begin{aligned}
D_{x_{k}}^{(\mu)}\left(x_{k} x^{u} y^{v}\right) & =\left(u_{k}+1\right) x^{u} y^{v}+\left(\sum_{j=1}^{r} y_{j} x_{k} \frac{\partial f_{j}^{(\mu)}}{\partial x_{k}}\right) x^{u} y^{v} \\
& =\left(u_{k}+1\right) x^{u} y^{v}+\sum_{j=1}^{r} \sum_{i=1}^{\delta_{j}} d_{j, i}(k) \mu_{j, i} x^{u+d_{j, i}} y^{v+e_{j}},
\end{aligned}
$$

thus $Z_{k}\left(\left[x^{u} y^{v}\right]_{\mu}\right)=-\left(u_{k}+1\right)\left[x^{u} y^{v}\right]_{\mu}$. For $k=1, \ldots, r$, we have

$$
\begin{aligned}
Z_{N+k}\left(\left[x^{u} y^{v}\right]_{\mu}\right) & =\left[\sum_{i=1}^{\delta_{k}} \mu_{k, i} D_{\mu_{k, i}}\left(x^{u} y^{v}\right)\right]_{\mu} \\
& =\left[\sum_{i=1}^{\delta_{k}} \mu_{k, i} x^{u+d_{k, i}} y^{v+e_{k}}\right]_{\mu} \\
& =\left[f_{k}^{(\mu)} x^{u} y^{v+e_{k}}\right]_{\mu} .
\end{aligned}
$$

But

$$
D_{y_{k}}^{(\mu)}\left(x^{u} y^{v+e_{k}}\right)=\left(v_{k}+1\right) x^{u} y^{v}+f_{k}^{(\mu)} x^{u} y^{v+e_{k}},
$$

thus $Z_{N+k}\left(\left[x^{u} y^{v}\right]_{\mu}\right)=-\left(v_{k}+1\right)\left[x^{u} y^{v}\right]_{\mu}$. This completes the proof of Theorem 6.4.

We use this result to describe left ideals in $D_{R}$ that annihilate the $\left[x^{u} y^{v}\right]$ and $\left[\omega_{u}\right]$. Let $I(u, v) \subseteq D_{\mathbf{C}[\mu]}$ be the left ideal generated by the $\square_{b}$ for $b \in E$, the $Z_{k}+u_{k}+1$ for $k=1, \ldots, N$, and the $Z_{N+k}+v_{k}+1$ for $k=1, \ldots, r$. By Theorem 6.4, I(u,v) annihilates $\left[x^{u} y^{v}\right]_{\mu}$. Let $\phi: \mathbf{C}[\mu] \rightarrow R$ be the homomorphism of C-algebras defined by setting $\phi\left(\mu_{j, i}\right)=\lambda_{j, i}$ for all $j, i$. Via $\phi$, we regard $R$ as a module over $\mathbf{C}[\mu]$. There is a standard construction that associates to any module $M$ over $D_{\mathbf{C}[\mu]}$ a module $M^{*}$ over $D_{R}$, namely, take 
$M^{*}=R \bigotimes_{\mathrm{C}[\mu]} M$ with the following action. We regard $M^{*}$ as a module over $R$ in the obvious way, while the action of $\partial \in \operatorname{Der}_{\mathbf{C}}(R)$ on $M^{*}$ is defined by the formula

$$
\partial(e \otimes m)=\partial(e) \otimes m+\sum_{j=1}^{r} \sum_{i=1}^{\delta_{j}} e \partial\left(\lambda_{j, i}\right) \otimes \frac{\partial}{\partial \mu_{j, i}}(m)
$$

for $e \in R$. This construction is functorial, in particular, the inclusion $\iota: I(u, v) \hookrightarrow D_{\mathbf{C}[\mu]}$ gives rise to a homomorphism of $D_{R}$-modules

$$
\iota^{*}=\text { id. } \otimes \iota: I(u, v)^{*}=R \bigotimes_{\mathbf{C}[\mu]} I(u, v) \rightarrow\left(D_{\mathbf{C}[\mu]}\right)^{*}=R \bigotimes_{\mathbf{C}[\mu]} D_{\mathbf{C}[\mu]} .
$$

Heuristically, $\left(D_{\mathbf{C}[\mu]}\right)^{*}$ consists of formal differential operators in the $\partial / \partial \mu_{j, i}$ with coefficients in $R$. Of course, these differential operators do not form a ring, in general, but they do form a module over $D_{R}$ (using the above definition). We define a homomorphism $\rho: D_{R} \rightarrow\left(D_{\mathbf{C}[\mu]}\right)^{*}$ of $D_{R}$-modules by setting $\rho(L)=L \cdot(1 \otimes 1)$. Let $I_{R}(u, v) \subseteq D_{R}$ be the inverse image of $\iota^{*}\left(I(u, v)^{*}\right)$ under $\rho$. It is a left ideal of $D_{R}$.

Theorem 6.7 The differential operators in the left ideal $I_{R}(u, v)$ annihilate the cohomology class $\left[x^{u} y^{v}\right] \in$ $H_{\mathrm{DR}}^{N+r}\left(C / R,\left(C, \nabla_{F}\right)\right)$.

The following corollary is then an immediate consequence of our discussion of $\left[\omega_{u}\right]$ at the beginning of this section.

Corollary 6.8 Suppose $f_{1}, \ldots, f_{r}$ define a smooth complete intersection $Y \subseteq \mathbf{A}_{R}^{N}$. Then the differential operators in the left ideal $I_{R}(u, 0)$ annihilate the cohomology class $\left[\omega_{u}\right] \in H_{\mathrm{DR}}^{N-r}(Y / S)$.

Proof of Theorem 6.7. Since $H_{\mathrm{DR}}^{N+r}\left(C / R,\left(C, \nabla_{F}\right)\right)$ is given by the right-hand side of (1.2), equation (6.5) and the right-exactness of tensor product give

$$
H_{\mathrm{DR}}^{N+r}\left(C / R,\left(C, \nabla_{F}\right)\right) \simeq R \bigotimes_{\mathbf{C}[\mu]} H_{\mathrm{DR}}^{N+r}\left(C_{\mu} / \mathbf{C}[\mu],\left(C_{\mu}, \nabla_{F^{(\mu)}}\right)\right)
$$

(an isomorphism of $D_{R}$-modules). One checks that under this isomorphism $\left[x^{u} y^{v}\right]$ corresponds to $1 \otimes\left[x^{u} y^{v}\right]_{\mu}$.

Let $\gamma_{u, v}: D_{\mathbf{C}[\mu]} \rightarrow H_{\mathrm{DR}}^{N+r}\left(C_{\mu} / \mathbf{C}[\mu],\left(C_{\mu}, \nabla_{F^{(\mu)}}\right)\right)$ be the homomorphism of $D_{\mathbf{C}[\mu]}$-modules defined by setting $\gamma_{u, v}(L)=L\left(\left[x^{u} y^{v}\right]_{\mu}\right)$. By Theorem 6.4, $I(u, v)$ lies in the kernel of $\gamma_{u, v}$. Tensoring with $R$, we get a homomorphism of $D_{R}$-modules

$$
\gamma_{u, v}^{*}:\left(D_{\mathbf{C}[\mu]}\right)^{*} \rightarrow R \bigotimes_{\mathbf{C}[\mu]} H_{\mathrm{DR}}^{N+r}\left(C_{\mu} / \mathbf{C}[\mu],\left(C_{\mu}, \nabla_{F^{(\mu)}}\right)\right)
$$

whose kernel contains $\iota^{*}\left(I(u, v)^{*}\right)$. Composing with $\rho$, gives a homomorphism of $D_{R}$-modules

$$
\gamma_{u, v}^{*} \circ \rho: D_{R} \rightarrow R \bigotimes_{\mathbf{C}[\mu]} H_{\mathrm{DR}}^{N+r}\left(C_{\mu} / \mathbf{C}[\mu],\left(C_{\mu}, \nabla_{F^{(\mu)}}\right)\right)
$$

which sends $L$ to $L \cdot\left(1 \otimes\left[x^{u} y^{v}\right]_{\mu}\right)$ and whose kernel contains $I_{R}(u, v)$. Using the identification (6.9), we may regard this as the homomorphism of $D_{R}$-modules from $D_{R}$ to $H_{\mathrm{DR}}^{N+r}\left(C / R,\left(C, \nabla_{F}\right)\right)$ that sends $L$ to $L\left(\left[x^{u} y^{v}\right]\right)$. The fact that its kernel contains $I_{R}(u, v)$ is the assertion of the theorem.

Example. Consider the Legendre family of (affine) elliptic curves $Y$ defined by $f\left(x_{1}, x_{2}\right)=0$, where

$$
f\left(x_{1}, x_{2}\right)=x_{2}^{2}-x_{1}\left(x_{1}-1\right)\left(x_{1}-\lambda\right) .
$$

We regard this as a smooth affine plane curve over $R=\mathbf{C}\left[\lambda,(\lambda(1-\lambda))^{-1}\right]$. The differential $\omega_{0} \in \Gamma\left(Y, \Omega_{Y / S}^{1}\right)$ is given on the open set where $x_{2} \neq 0$ by $\omega_{0}=d x_{1} / 2 x_{2}$. We show that the left ideal $I_{R}(0,0)$, which by Corollary 6.8 annihilates $\left[\omega_{0}\right] \in H_{\mathrm{DR}}^{1}(Y / S)$, is generated by the Gaussian hypergeometric operator

$$
\left(\frac{d}{d \lambda}\right)^{2}+\frac{1-2 \lambda}{\lambda(1-\lambda)} \frac{d}{d \lambda}-\frac{1}{4 \lambda(1-\lambda)}
$$


Multiplying out the formula for $f\left(x_{1}, x_{2}\right)$, we get

$$
f\left(x_{1}, x_{2}\right)=x_{2}^{2}-x_{1}^{3}+(\lambda+1) x_{1}^{2}-\lambda x_{1} .
$$

Thus

$$
f^{(\mu)}\left(x_{1}, x_{2}\right)=\mu_{1} x_{2}^{2}+\mu_{2} x_{1}^{3}+\mu_{3} x_{1}^{2}+\mu_{4} x_{1}
$$

and the map $\phi: \mathbf{C}[\mu] \rightarrow R$ is defined by

$$
\phi\left(\mu_{1}\right)=1, \quad \phi\left(\mu_{2}\right)=-1, \quad \phi\left(\mu_{3}\right)=\lambda+1, \quad \phi\left(\mu_{4}\right)=-\lambda .
$$

By definition, the left ideal $I(0,0) \subseteq D_{\mathbf{C}[\mu]}$ is generated by the four operators $Z_{i}+1$ for $i=1,2,3$, where

$$
\begin{aligned}
& Z_{1}=3 \mu_{2} \frac{\partial}{\partial \mu_{2}}+2 \mu_{3} \frac{\partial}{\partial \mu_{3}}+\mu_{4} \frac{\partial}{\partial \mu_{4}} \\
& Z_{2}=2 \mu_{1} \frac{\partial}{\partial \mu_{1}} \\
& Z_{3}=\mu_{1} \frac{\partial}{\partial \mu_{1}}+\mu_{2} \frac{\partial}{\partial \mu_{2}}+\mu_{3} \frac{\partial}{\partial \mu_{3}}+\mu_{4} \frac{\partial}{\partial \mu_{4}},
\end{aligned}
$$

and

$$
\square=\left(\frac{\partial}{\partial \mu_{3}}\right)^{2}-\frac{\partial}{\partial \mu_{2}} \frac{\partial}{\partial \mu_{4}} .
$$

There is a natural map $D_{\mathbf{C}[\mu]} \rightarrow\left(D_{\mathbf{C}[\mu]}\right)^{*}$ which sends $L$ to $1 \otimes L$. To simplify notation, we write $L^{*}$ in place of $1 \otimes L$ and we represent elements of $\left(D_{\mathbf{C}[\mu]}\right)^{*}$ as differential operators in the $\partial / \partial \mu_{i}$ with coefficients in $R$. Thus if

$$
L=\sum_{k} g_{k}\left(\mu_{1}, \mu_{2}, \mu_{3}, \mu_{4}\right)\left(\frac{\partial}{\partial \mu}\right)^{k} \in D_{\mathbf{C}[\mu]},
$$

where $k$ is a multi-index, then

$$
L^{*}=\sum_{k} g_{k}(1,-1, \lambda+1,-\lambda)\left(\frac{\partial}{\partial \mu}\right)^{k} \in\left(D_{\mathbf{C}[\mu]}\right)^{*} .
$$

The $D_{R}$-homomorphism $\rho: D_{R} \rightarrow\left(D_{\mathbf{C}[\mu]}\right)^{*}$ can then be expressed as follows. If $P=\sum_{i=1}^{n} h_{i}(\lambda)(\partial / \partial \lambda)^{i} \in$ $D_{R}$, then

$$
\rho(P)=\sum_{i=1}^{n} h_{i}(\lambda)\left(\frac{\partial}{\partial \mu_{3}}-\frac{\partial}{\partial \mu_{4}}\right)^{i} \in\left(D_{\mathbf{C}[\mu]}\right)^{*} .
$$

The left ideal $I_{R}(0,0) \subseteq D_{R}$ consists of all $P \in D_{R}$ such that $\rho(P) \in \iota^{*}\left(I(0,0)^{*}\right)$.

Lemma 6.13 The $D_{R}$-module $\left(D_{\mathbf{C}[\mu]}\right)^{*} / \iota^{*}\left(I(0,0)^{*}\right)$ is a free, rank $2 R$-module with basis $1, \partial / \partial \mu_{3}$.

Proof. The fact that $Z_{i}+1 \in I(0,0)$ for $i=1,2,3$ allows us to express $\mu_{i} \partial / \partial \mu_{i}$ modulo $I(0,0)$ for $i=1,2,4$ in terms of $\mu_{3} \partial / \partial \mu_{3}$. Explicitly,

$$
\begin{aligned}
\mu_{1} \frac{\partial}{\partial \mu_{1}} & \equiv-\frac{1}{2} \quad(\bmod I(0,0)) \\
\mu_{2} \frac{\partial}{\partial \mu_{2}} & \equiv \mu_{4} \frac{\partial}{\partial \mu_{4}} \quad(\bmod I(0,0)) \\
\mu_{4} \frac{\partial}{\partial \mu_{4}} & \equiv-\frac{\mu_{3}}{2} \frac{\partial}{\partial \mu_{3}}-\frac{1}{4} \quad(\bmod I(0,0)) .
\end{aligned}
$$

It follows from these equations that, after multiplication by a monomial in $\mu_{1}, \mu_{2}, \mu_{4}$, every $L \in D_{\mathbf{C}[\mu]}$ can be expressed modulo $I(0,0)$ as a polynomial in $\partial / \partial \mu_{3}$ with coefficients in $\mathbf{C}[\mu]$. Since $\lambda$ is invertible in $R$, this implies that the powers of $\partial / \partial \mu_{3} \operatorname{span}\left(D_{\mathbf{C}[\mu]}\right)^{*} / \iota^{*}\left(I(0,0)^{*}\right)$ as $R$-module. From (6.11) we get

$$
\left(\mu_{2} \mu_{4}\right) \square=\mu_{2} \mu_{4}\left(\frac{\partial}{\partial \mu_{3}}\right)^{2}-\left(\mu_{2} \frac{\partial}{\partial \mu_{2}}\right)\left(\mu_{4} \frac{\partial}{\partial \mu_{4}}\right) \in I(0,0) .
$$


Substitution from (6.15) and (6.16) then gives

$$
\left(4 \mu_{2} \mu_{4}-\mu_{3}^{2}\right)\left(\frac{\partial}{\partial \mu_{3}}\right)^{2}-2 \mu_{3} \frac{\partial}{\partial \mu_{3}}-\frac{1}{4} \in I(0,0) .
$$

This implies that

$$
\left(\frac{\partial}{\partial \mu_{3}}\right)^{2}+\frac{2(\lambda+1)}{(\lambda-1)^{2}} \frac{\partial}{\partial \mu_{3}}+\frac{1}{4(\lambda-1)^{2}} \in \iota^{*}\left(I(0,0)^{*}\right)
$$

and also that $1, \partial / \partial \mu_{3} \operatorname{span}\left(D_{\mathbf{C}[\mu]}\right)^{*} / \iota^{*}\left(I(0,0)^{*}\right)$ as $R$-module. If there were a nontrivial $R$-linear relation between 1 and $\partial / \partial \mu_{3}$ in this quotient, it would follow from (6.16) that there is also a nontrivial $R$-linear relation between 1 and $\partial / \partial \mu_{3}-\partial / \partial \mu_{4}$. By (6.12), this would imply that there exists a nontrivial first-order operator in $I_{R}(0,0)$. Such an operator annihilates $\left[\omega_{0}\right]$, hence there is a nontrivial $R$-linear relation between $\left[\omega_{0}\right]$ and $(\partial / \partial \lambda)\left(\left[\omega_{0}\right]\right)$. But it is well-known (and easily checked) that

$$
\frac{\partial}{\partial \lambda}\left(\left[\omega_{0}\right]\right)=-\frac{1}{2(\lambda-1)}\left[\omega_{0}\right]+\frac{1}{2 \lambda(\lambda-1)}\left[\omega_{1}\right]
$$

giving a nontrivial $R$-linear relation between $\left[\omega_{0}\right]$ and $\left[\omega_{1}\right]$. But this contradicts the fact that $\left[\omega_{0}\right]$ and $\left[\omega_{1}\right]$ form a basis for the de Rham cohomology of the generic fiber of $Y \rightarrow S$. This establishes the lemma.

We now consider a second-order operator

$$
P=\left(\frac{d}{d \lambda}\right)^{2}+A(\lambda) \frac{d}{d \lambda}+B(\lambda) \in D_{R}
$$

for which we have

$$
\rho(P)=\left(\frac{\partial}{\partial \mu_{3}}-\frac{\partial}{\partial \mu_{4}}\right)^{2}+A(\lambda)\left(\frac{\partial}{\partial \mu_{3}}-\frac{\partial}{\partial \mu_{4}}\right)+B(\lambda) \in\left(D_{\mathbf{C}[\mu]}\right)^{*} .
$$

We express $\rho(P)$ modulo $\iota^{*}\left(I(0,0)^{*}\right)$ in terms of the basis $1, \partial / \partial \mu_{3}$. The procedure is to expand the expression (6.21) and express $\left(\partial / \partial \mu_{4}\right)^{2},\left(\partial / \partial \mu_{3}\right)\left(\partial / \partial \mu_{4}\right)$, and $\partial / \partial \mu_{4}$ in terms of $1, \partial / \partial \mu_{3}$, and $\left(\partial / \partial \mu_{3}\right)^{2}$. We can then use (6.19) to express everything in terms of 1 and $\partial / \partial \mu_{3}$. Using (6.15) in (6.11) gives

$$
\mu_{2}\left(\frac{\partial}{\partial \mu_{3}}\right)^{2}-\mu_{4}\left(\frac{\partial}{\partial \mu_{4}}\right)^{2}-\frac{\partial}{\partial \mu_{4}} \in I(0,0)
$$

hence

$$
\left(\frac{\partial}{\partial \mu_{4}}\right)^{2} \equiv \frac{1}{\lambda}\left(\frac{\partial}{\partial \mu_{3}}\right)^{2}+\frac{1}{\lambda} \frac{\partial}{\partial \mu_{4}} \quad\left(\bmod \iota^{*}\left(I(0,0)^{*}\right)\right) .
$$

Applying $\partial / \partial \mu_{3}$ to $(6.16)$ gives

$$
2 \mu_{4} \frac{\partial}{\partial \mu_{3}} \frac{\partial}{\partial \mu_{4}}+\mu_{3}\left(\frac{\partial}{\partial \mu_{3}}\right)^{2}+\frac{3}{2} \frac{\partial}{\partial \mu_{3}} \in I(0,0)
$$

hence

$$
\frac{\partial}{\partial \mu_{3}} \frac{\partial}{\partial \mu_{4}} \equiv \frac{\lambda+1}{2 \lambda}\left(\frac{\partial}{\partial \mu_{3}}\right)^{2}+\frac{3}{4 \lambda} \frac{\partial}{\partial \mu_{3}} \quad\left(\bmod \iota^{*}\left(I(0,0)^{*}\right)\right) .
$$

Equation (6.16) gives

$$
\frac{\partial}{\partial \mu_{4}} \equiv \frac{\lambda+1}{2 \lambda} \frac{\partial}{\partial \mu_{3}}+\frac{1}{4 \lambda} \quad\left(\bmod \iota^{*}\left(I(0,0)^{*}\right)\right)
$$

Expanding (6.21) and substituting (6.22), (6.23), and (6.24) gives

$$
\rho(P) \equiv\left(\frac{1-2 \lambda}{2 \lambda^{2}}+A(\lambda) \frac{\lambda-1}{2 \lambda}\right) \frac{\partial}{\partial \mu_{3}}+\frac{1}{4 \lambda^{2}}-A(\lambda) \frac{1}{4 \lambda}+B(\lambda) \quad\left(\bmod \iota^{*}\left(I(0,0)^{*}\right)\right) .
$$


Solving for $A(\lambda)$ and $B(\lambda)$, we see that $\rho(P) \equiv 0\left(\bmod \iota^{*}\left(I(0,0)^{*}\right)\right)$ if and only if

$$
A(\lambda)=\frac{1-2 \lambda}{\lambda(1-\lambda)} \quad \text { and } \quad B(\lambda)=-\frac{1}{4 \lambda(1-\lambda)} .
$$

Substituting these expressions in (6.20) gives (6.10).

We have proved that (6.10) is the unique monic second-order operator in $I_{R}(0,0)$. We saw in the proof of Lemma 6.13 that there are no lower-order operators in $I_{R}(0,0)$, hence $(6.10)$ generates this left ideal.

\section{References}

[1] A. Adolphson, Hypergeometric functions and rings generated by monomials, Duke Math. J. 73 (1994), 269-290

[2] A. Adolphson and S. Sperber, On the zeta function of a complete intersection, Ann. Sci. E. N. S. 29 (1996), 287-328

[3] A. Adolphson and S. Sperber, Differential modules defined by systems of equations, Rend. Sem. Mat. Univ. Padova 95 (1996), 37-57

[4] A. Altman and S. Kleiman, Introduction to Grothendieck Duality Theory, Lecture Notes in Math. no. 146, Springer-Verlag, Berlin-Heidelberg-New York, 1970

[5] V. V. Batyrev, Variations of the mixed Hodge structure of affine hypersurfaces in algebraic tori, Duke Math. J. 69 (1993), 349-409

[6] P. Deligne, Equations Différentielles à Points Singuliers Réguliers, Lecture Notes in Math. no. 163, Springer-Verlag, Berlin-Heidelberg-New York, 1970

[7] A. Dimca, F. Maaref, C. Sabbah, and M. Saito, Dwork cohomology and algebraic $\mathcal{D}$-modules, preprint (math.AG/9906078)

[8] B. Dwork, On the zeta function of a hypersurface, II, Ann. of Math. 80 (1964), 227-299

[9] B. Dwork, Generalized Hypergeometric Functions, Oxford University Press, New York, 1990

[10] B. Dwork and F. Loeser, Hypergeometric series, Japan. J. Math. 19 (1993), 81-129

[11] I. M. Gelfand, A. V. Zelevinskii, and M. M. Kapranov, Hypergeometric functions and toral manifolds, English translation, Functional Anal. Appl. 23 (1989), 94-106

[12] R. Hartshorne, Ample Subvarieties of Algebraic Varieties, Lecture Notes in Math. no. 156, SpringerVerlag, Berlin-Heidelberg-New York, 1970

[13] N. Katz, Thesis, Princeton University (1966)

[14] N. Katz, On the differential equations satisfied by period matrices, Publ. Math. I. H. E. S. 35 (1968), $223-258$

[15] N. Katz, Nilpotent connections and the monodromy theorem: applications of a result of Turrittin, Publ. Math. I. H. E. S. 39 (1971), 175-232

[16] E. Kunz, Introduction to Commutative Algebra and Algebraic Geometry, Birkhäuser, Boston, 1985

[17] H. Matsumura, Commutative Ring Theory, Cambridge University Press, Cambridge, 1986

[18] P. Monsky, p-Adic Analysis and Zeta Functions, Lectures in Mathematics, Kyoto University, Kinokuniya Bookstore

[19] K. Saito, On a generalization of de Rham lemma, Ann. Inst. Fourier, Grenoble 26 (1976), 165-170 